\title{
Intermittent hemodialysis in dogs with chronic kidney disease stage III
}

\author{
Alessandra Melchert ${ }^{1}$ Silvano Salgueiro Geraldes ${ }^{1}$ André Nanny Le Sueur Vieira ${ }^{1}$ \\ Regina Kiomi Takahira ${ }^{1}$ Paulo Roberto Rodrigues Ramos ${ }^{2}$ Pasqual Barretti ${ }^{3}$ \\ Carlos Roberto Padovani ${ }^{2}$ Paula Bilbau Sant'Ana ${ }^{1}$ José Francisco Antunes Ribeiro ${ }^{1}$ \\ Priscylla Tatiana Chalfun Guimarães-Okamoto ${ }^{1 *}$
}

${ }^{1}$ Departamento de Clínica Veterinária, Faculdade de Medicina Veterinária e Zootecnia (FMVZ), Universidade Estadual Paulista (UNESP), 18618-681, Botucatu, SP, Brasil. E-mail: tatiana@fmvz.unesp.br. "Corresponding author.

${ }^{2}$ Departamento de Física, Biofísica e Bioestatística, Instituto de Biociências (IBB), Universidade Estadual Paulista (UNESP), Botucatu, SP, Brasil. ${ }^{3}$ Departamento de Clínica Médica, Faculdade de Medicina (FMB), Universidade Estadual Paulista (UNESP), Botucatu, SP, Brasil.

ABSTRACT: Intermittent hemodialysis (IHD) is a form of renal replacement that is used in veterinary medicine for cases involving drug removal, electrolyte imbalance, acute kidney injury, and chronic kidney disease (CKD). The aim of the present study was to verify the efficacy of IHD in dogs with CKD staged at grade III and to evaluate the effect of IHD on quality of life. Twelve dogs with CKD at stage III met the inclusion criteria and were divided equally into two groups. The control group $(n=6)$ received only clinical treatment and intravenous fluid therapy, and the hemodialysis group $(n=6)$ received clinical and IHD treatments. Blood samples were collected before and after treatments in both groups. We evaluated complications and clinical parameters of IHD every 30 minutes. Hemodialysis decreased serum urea, creatinine, and phosphorus. Despite the evident removal of nitrogen compounds, dialysis treatment did not increase survival time in these patients. The results of this study do not support the early use of dialysis in dogs with chronic kidney disease stage III.

Key words: dialysis, $C K D$, kidney, nephrology, dog.

Hemodiálise intermitente em cães com doença renal crônica III

RESUMO: A Hemodiálise Intermitente (HDI) é uma modalidade de substituição renal, utilizada na veterinária nos casos de remoção de drogas, distúrbios hidroeletrolitico, lesão renal aguda e doença renal crônica (DRC). O objetivo do presente estudo foi estudar o efeito da hemodiálise intermitente em cães com DRC estadeados no grau III, visando avaliar a qualidade de vida. Foram selecionados 12 cães com DRC no estádio III pelos critérios de inclusão, após foram divididos em Grupo Controle ( $n=6)$, onde foi preconizado apenas tratamento clínico e fluidoterapia, e Grupo hemodiálise (n=6), que além do tratamento clínico, utilizou-se a hemodiálise intermitente. A colheita de sangue para avaliação laboratorial foi realizada antes e após a fluidoterapia de ambos os grupos. Foram avaliados as intercorrências e os parâmetros clínicos durante a HDI a cada 30 minutos. A instituição do tratamento dialítico promoveu uma eficaz diminuição das concentrações séricas de ureia, creatinina e fósforo de modo mais precoce. Apesar da evidente remoção dos compostos nitrogenados, o tratamento dialítico não aumentou a sobrevida destes pacientes, não justificando o emprego desta técnica de forma precoce em cães com doença renal crônica no estádio III.

Palavras-chave: diálise, $D R C$, rim, nefrologia, cão.

\section{INTRODUCTION}

Chronic kidney disease (CKD) is a commonly diagnosed disease in small animal practice. It is a progressive disease that causes functional alterations in the renal parenchyma, which may ultimately progress to renal failure (POLZIN, 2011). CKD can be diagnosed through history, clinical findings, laboratory tests, imaging and by kidney biopsy (POLZIN, 2013; WAKI et al., 2015). CKD can affect all ages; it may occur as an acquired disease in elderly patients or as a congenital or heritable form in juvenile animals (BARTAGES, 2012). Dogs with CKD in stage III present moderate azotemia with serum creatinine of $2.1-5.0 \mathrm{mg} \mathrm{dl}^{-1}$, and its clinical manifestations at this stage are more pronounced, requiring close monitoring and patient care (POLZIN, 2013).

Intermittent hemodialysis (IHD) is an extracorporeal renal replacement therapy that involves relatively short hemodialysis sessions (BLOOM \& LABATO, 2011). IHD treatment for humans with $\mathrm{CKD}$ is intended to reduce azotemia, correct fluid and electrolyte imbalances, and give support to CKD patients in stage $\mathrm{V}$ before kidney transplantation. The IHD treatment for dogs may be indicated for drug and 
toxin removal, electrolyte imbalances, acute renal failure (ARF), and acute exacerbation of CKD (COWGILL, 2011). The prognosis and therapy time differ for each patient depending on the underlying cause of disease, degree of renal injury, and presence of comorbidities (FISCHER et al., 2004). IHD has been used in veterinary medicine for 40 years, showing scientific evidence that it can be a safe and effective technique when performed correctly (COWGILL \& GUILLAUMIN, 2013).

The aim of this study was to evaluate the effect of intermittent hemodialysis in dogs with CKD stage III and to compare the results with a control group of dogs in the same stage receiving intravenous fluid therapy prescription as main treatment.

\section{MATERIALS AND METHODS}

The study included 12 dogs with CKD stage III randomly assigned to two groups. Dogs were of both sexes, and the mean weight and age of the control group (CG) and hemodialysis group (HDG) were $19.53 \pm 8.12 \mathrm{~kg}$ and $10.00 \pm 4.14$ years and $16.59 \pm 5.42 \mathrm{~kg}$ and $8.83 \pm 2.71$ years, respectively. The CG $(n=6)$ and the HDG $(n=6)$ received clinical treatment and HDG also underwent two IHD sessions per week.

The first IHD session lasted for 1 hour, and subsequent sessions lasted for 120 minutes. Despite the advanced stage of CKD associated with the progressive loss of serum albumin and lower hematocrit levels, the dialysis time was reduced to 120 minutes, respecting the treatment intensity prescription of URR (COWGILL, 2011). Both treatments (hemodialysis and fluid therapy) were performed twice a week for 60 days.

Per the inclusion criteria, the dogs were evaluated for 60 days, including laboratory analysis of serum urea, creatinine, systolic blood pressure (SBP), and urinary protein-creatinine ratio $(\mathrm{UP} / \mathrm{C})$. When CKD was confirmed, staging was done according to the methodology of the International Renal Interest Society (IRIS) (IRIS, 2015). During randomized selection, the exclusion criteria were acute kidney injury on $\mathrm{CKD}$, hypovolemia, coagulation disorders, laboratorial signs of infection, pancreatitis, heart failure, nephrolithiasis, and neoplasia.

The clinical treatment for both groups consisted of intravenous fluid therapy with an isotonic polyionic solution twice a week. HDG received fluid therapy treatment due to the dialytic principles of convection and minimal ultrafiltration (COWGILL \& FRANCEY, 2012). Dehydration was corrected according to the basic principles of fluid therapy (DIBARTOLA \& BATEMAN, 2012). Nausea and vomiting were controlled with the administration of a proton pump blocker and antiemetic drugs. Human recombinant erythropoietin was prescribed to correct anemia when hematocrit was $\leq 15 \%$, always combined with ferrous sulfate supplementation. Antihypertensive treatment was prescribed with an angiotensin-converting enzyme inhibitor (ACEI) as monotherapy or combined with a calcium channel blocker (CCB) when needed. Also, aluminum hydroxide to control phosphorus levels along with a prescription kidney diet was prescribed to all patients.

Dogs subjected to hemodialysis were catheterized with a double-lumen catheter in the right or left external jugular. The HDI was performed with a 4008S Fresenius Hemodialysis System, and the hemodialyzer had a surface area of $0.8 \mathrm{~m}^{2}$ and was primed with $52 \mathrm{ml}$ (Hemoflow F4, Fresenius Medical Care). IHD aimed to reach values of predialysis serum urea of $150 \mathrm{mg} \mathrm{dL}^{-1}$ (BUN, 70mg dL $\mathrm{m}^{-1}$ ) and postdialysis serum urea of $21.4 \mathrm{mg} \mathrm{dL}^{-1}$ (BUN, $10 \mathrm{mg} \mathrm{dL}^{-1}$ ), according to the report of COWGILL \& FRANCEY (2012).

Blood samples were collected from both groups, and they received clinical, laboratorial, and physical evaluations. For both groups, two blood samples were collected on the treatment day: one sample was collected before the clinical treatment or hemodialysis (PRE), and the other sample (POST) was collected immediately after the end of fluid therapy in the control group and 60 minutes after the end of treatment for IHD, avoiding recirculation withdrawn. Serum urea, creatinine, albumin, calcium, phosphorus, sodium, and potassium were also assessed. Dogs were evaluated on Day 1 and at the following treatment sessions: Day 5, Day 8, Day 12, and Day 15.

Data were analyzed statistically, considering various situations. Comparisons between POST exam tests for the two groups and values obtained from PRE and POST exams for each group at each selected treatment session were compared using the Kolmogorov-Smirnov test. Clinical data such as hypertension, incidence of vomiting, and appetite were analyzed and compared using descriptive methods and occurrence percentages. A 5\% level of significance was applied (JOHNSON \& WICHERN, 2001). Survival curves were computed to determine the percentage of surviving animals during the observation period. The Gehan-Breslow-Wilcoxon test was used to compare survival rates between the CG and HDG groups. Statistical analyses were performed using GraphPad Prism 6.0.

\section{RESULTS AND DISCUSSION}

Generally, prescriptions of IHD for dogs with CKD aims to promote a better quality of life than 
to reduce the acute uremia signs most seen and reported in the acute phase (COWGILL \& FRANCEY, 2012). In the present study, although IHD was more efficient at removing nitrogenous end products from the blood, the subsequent morbidities that present along with CKD may have implications for the survival of the patient. The higher the stage, the more difficult it is to control morbidities along with CKD. Further studies are needed due to the short period of analysis, number of patients, and a better dialysis time adequacy.

Hypertension in patients with CKD may be related to defective sodium excretion in the kidney, increased production of vasoconstrictors such as angiotensin II, decreased vasodilators such as prostaglandins, and changes in endothelial function with impaired nitric oxide synthesis (COWGILL \& FRANCEY, 2012).

Hypertension was observed in $33.33 \%$ of the CG and $66.6 \%$ of the HDG at the start of the study. There was a slight reduction in the percentage of hypertensive patients throughout the study in both groups due to antihypertensive treatment with ACEI and $\mathrm{CCB}$. In contrast, hypotension is the most serious complication that can occur near to the end of the hemodialysis session due to loss of the blood volume at the beginning of extracorporeal circulation or due to cytokines and other inflammatory mediators from the interaction of blood with the dialysis membrane (KOMENO et al., 2004). In two hemodialysis sessions, only one $\operatorname{dog}(8 \%)$ had hypotension $(80 \mathrm{mmHg})$.

Multiple factors contribute to anemia in dogs receiving hemodialysis (LANGSTON et al., 2017). Hematological evaluation of the red blood cells (RBC), hematocrit (Ht), and hemoglobin $(\mathrm{Hb})$ in $\mathrm{CG}$ dogs showed reduced values in the POST samples from Days 1 to 8 and Day 15 , but only the $\mathrm{Ht}$ and $\mathrm{Hb}$ values from Day 8 were statistically significant (Table 1).

In $\mathrm{HDG}, \mathrm{RBC}, \mathrm{Ht}$, and $\mathrm{Hb}$ were lower in POST samples from all tested days despite blood loss that occurred due to the extracorporeal procedure, but only the reduction in $\mathrm{Ht}$ values from Days 1,8, and 12 was statistically significant (Table 1). Comparisons of these variables did not reveal differences between groups (Table 2). There were sharp declines in RBC, $\mathrm{Ht}$, and $\mathrm{Hb}$ values during the HDG; although, these differences were not statistically significant.

Anemia is a common complication in CKD due to deficiencies of erythropoietin and iron (FALCO et al., 2013). In HDG, blood loss during dialysis reduces

Table 1 - Clinical values (average \pm standard deviation) of red blood cell (RBC) count $\left(\times 10^{6} \mu \mathrm{L}^{-1}\right)$, hematocrit $(\%)$, hemoglobin, and total platelet count $\left(\times 10^{3} \mathrm{~mm}^{-3}\right)$ of animals in the control group (CG) and the hemodialysis group (HDG) before (PRE) and after (POST) treatment (Day 1 to Day 15).

\begin{tabular}{|c|c|c|c|c|c|}
\hline Parameters & Day1 & Day 5 & Day 8 & Day 12 & Day 15 \\
\hline \multicolumn{6}{|c|}{${ }$} \\
\hline CG Pre & $4.62 \pm 1.31^{\mathrm{a}}$ & $4.42 \pm 1.27^{\mathrm{a}}$ & $4.23 \pm 1.49^{\mathrm{a}}$ & $4.19 \pm 1.42^{\mathrm{a}}$ & $4.51 \pm 1.80^{\mathrm{a}}$ \\
\hline CG Post & $4.27 \pm 1.59^{\mathrm{a}}$ & $4.20 \pm 0.59^{\mathrm{a}}$ & $3.95 \pm 1.77^{\mathrm{a}}$ & $4.29 \pm 1.34^{\mathrm{a}}$ & $4.30 \pm 2.04^{\mathrm{a}}$ \\
\hline HDG Pre & $5.89 \pm 3.86^{\mathrm{a}}$ & $3.67 \pm 1.08^{\mathrm{a}}$ & $3.83 \pm 0.78^{\mathrm{a}}$ & $3.65 \pm 0.97^{\mathrm{a}}$ & $2.76 \pm 0.35^{\mathrm{a}}$ \\
\hline HDG Post & $3.72 \pm 1.35^{\mathrm{a}}$ & $3.44 \pm 1.09^{\mathrm{a}}$ & $3.45 \pm 1.01^{\mathrm{a}}$ & $3.21 \pm 0.92^{\mathrm{a}}$ & $2.47 \pm 0.61^{\mathrm{a}}$ \\
\hline \multicolumn{6}{|c|}{ 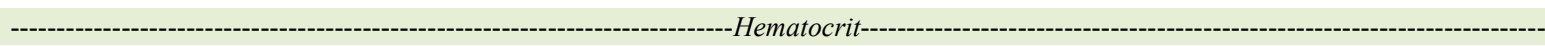 } \\
\hline CG Pre & $32.16 \pm 7.96^{\mathrm{a}}$ & $30.50 \pm 7.23^{\mathrm{a}}$ & $28.66 \pm 10.28^{\mathrm{a}^{*}}$ & $29.50 \pm 9.31^{\mathrm{a}}$ & $31.00 \pm 11.59^{\mathrm{a}}$ \\
\hline CG Post & $29.60 \pm 10.59^{\mathrm{a}}$ & $27.66 \pm 7.42^{\mathrm{a}}$ & $27.20 \pm 12.02^{b}$ & $30.40 \pm 8.62^{\mathrm{a}}$ & $30.00 \pm 12.98^{a}$ \\
\hline HDG Pre & $28.83 \pm 10.81^{\mathrm{a}^{*}}$ & $25.50 \pm 8.26^{\mathrm{a}}$ & $27.20 \pm 6.01^{\mathrm{a}^{*}}$ & $24.80 \pm 2.95^{\mathrm{a}^{*}}$ & $18.66 \pm 2.88^{\mathrm{a}}$ \\
\hline HDG Post & $25.33 \pm 8.98^{\mathrm{b}}$ & $23.83 \pm 8.99^{\mathrm{a}}$ & $23.20 \pm 6.94^{\mathrm{b}}$ & $22.20 \pm 2,74^{\mathrm{b}}$ & $16.66 \pm 3.78^{\mathrm{a}}$ \\
\hline \multicolumn{6}{|c|}{ - } \\
\hline CG Pre & $11.10 \pm 3.58^{\mathrm{a}^{*}}$ & $10.53 \pm 2.86^{\mathrm{a}}$ & $10.15 \pm 3.79^{\mathrm{a}^{*}}$ & $9.95 \pm 3.43^{\mathrm{a}}$ & $10.68 \pm 4.37^{\mathrm{a}}$ \\
\hline CG Post & $10.18 \pm 4.05^{\mathrm{b}}$ & $10.01 \pm 3.55^{\mathrm{a}}$ & $9.64 \pm 4.27^{\mathrm{b}}$ & $10.66 \pm 3.40^{\mathrm{a}}$ & $10.38 \pm 4.90^{\mathrm{a}}$ \\
\hline HDG Pre & $9.81 \pm 3.89^{\mathrm{a}^{*}}$ & $8.75 \pm 3.00^{\mathrm{a}}$ & $9.12 \pm 1.90^{\mathrm{a}}$ & $8.36 \pm 2.20^{\mathrm{a}}$ & $6.26 \pm 0.89^{\mathrm{a}}$ \\
\hline HDG Post & $8.76 \pm 3.39^{\mathrm{b}}$ & $8.15 \pm 2.79^{\mathrm{a}}$ & $7.90 \pm 2.64 b$ & $7.54 \pm 2.12^{\mathrm{a}}$ & $5.66 \pm 1.50^{\mathrm{a}}$ \\
\hline \multicolumn{6}{|c|}{ 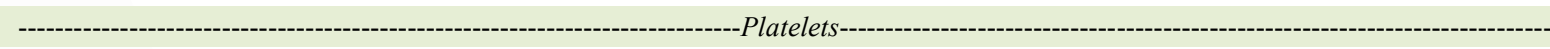 } \\
\hline CG Pre & $238333 \pm 95511^{\mathrm{a}}$ & $266167 \pm 120617^{\mathrm{a}}$ & $291667 \pm 124596^{\mathrm{a}}$ & $289304 \pm 140550^{\mathrm{a}}$ & $267485 \pm 165246^{\mathrm{a}}$ \\
\hline CG Post & $212600 \pm 73819^{\mathrm{a}}$ & $255167 \pm 92500^{\mathrm{a}}$ & $270250 \pm 117236^{\mathrm{a}}$ & $299500 \pm 161544^{\mathrm{a}}$ & $269000 \pm 195095^{\mathrm{a}}$ \\
\hline HDG Pre & $210833 \pm 64502^{\mathrm{a}}$ & $195163 \pm 32425^{\mathrm{a}^{*}}$ & $177160 \pm 92706^{\mathrm{a}}$ & $254500 \pm 107730^{\mathrm{a}}$ & $241667 \pm 148514^{\mathrm{a}}$ \\
\hline HDG Post & $145358 \pm 33102^{\mathrm{a}}$ & $140150 \pm 59512^{b}$ & $131120 \pm 71682^{\mathrm{a}}$ & $227795 \pm 157929^{a}$ & $185808 \pm 119675^{\mathrm{a}}$ \\
\hline
\end{tabular}

Different letters represent statistically significant differences over time in the same treatment; ${ }^{*} P<0.05 \times$ Post; ${ }^{* *} P<0.01 \mathrm{x}$ Post. 
Table 2 - Clinical values (average \pm standard deviation) of red blood cell $(\mathrm{RBC})$ count $\left(\times 10^{6} \mu \mathrm{L}^{-1}\right)$, hematocrit $(\%)$, hemoglobin, total platelet count $\left(\times 10^{3} \mathrm{~mm}^{-3}\right)$, total leukocyte count $\left(\times 10^{3} \mathrm{~mm}^{-3}\right)$, serum urea $\left(\mathrm{mg} \mathrm{dL}^{-1}\right)$, creatinine $\left(\mathrm{mg} \mathrm{dL}^{-1}\right)$, albumin $\left(\mathrm{g} \mathrm{dL} \mathrm{Cl}^{-1}\right)$, phosphorus $\left(\mathrm{mg} \mathrm{dL}^{-1}\right)$, ionic calcium $\left(\mathrm{mmol} \mathrm{L}^{-1}\right)$ and potassium $\left(\mathrm{mEq} \mathrm{L}^{-1}\right)$ of animals in the control group (CG) after clinical treatment with intravenous fluid therapy (Post-C) and animals in the hemodialysis group (HDG) after HD sessions (Post-HD) from Day 1 to Day 15.

\begin{tabular}{|c|c|c|c|c|c|c|c|c|c|c|c|}
\hline Groups & & RBC's & $\mathrm{Ht}$ & $\mathrm{Hb}$ & Platelets & Urea & $\mathrm{sCr}$ & sAlb & $\mathrm{P}$ & $\mathrm{iCa}^{+2}$ & $\mathrm{~K}^{+}$ \\
\hline \multirow{2}{*}{ Day 1} & Post-C & $\begin{array}{c}4.27 \pm 1 \\
59^{\mathrm{a}}\end{array}$ & $\begin{array}{c}29.60 \pm \\
10.59^{\mathrm{a}}\end{array}$ & $\begin{array}{c}10.18 \pm \\
4.05^{\mathrm{a}}\end{array}$ & $\begin{array}{c}212600 \pm \\
73819^{\mathrm{a}}\end{array}$ & $\begin{array}{c}174.43 \pm \\
56.96^{\mathrm{a}}\end{array}$ & $\begin{array}{l}4.04 \pm \\
0.78^{\mathrm{a}^{*}}\end{array}$ & $\begin{array}{c}2.31 \pm \\
0.24^{\mathrm{a}}\end{array}$ & $\begin{array}{l}6.86 \pm \\
2.38^{\mathrm{a}}\end{array}$ & $\begin{array}{l}0.764 \pm \\
0.092^{\mathrm{a}}\end{array}$ & $\begin{array}{l}4.06 \pm \\
0.59^{\mathrm{a}}\end{array}$ \\
\hline & Post-HD & $\begin{array}{c}3.72 \pm 1 \\
35^{\mathrm{a}}\end{array}$ & $\begin{array}{c}25.33 \pm \\
8.98^{\mathrm{a}}\end{array}$ & $\begin{array}{l}8.76 \pm \\
3.39^{\mathrm{a}}\end{array}$ & $\begin{array}{c}145358 \pm \\
33102^{\mathrm{a}}\end{array}$ & $\begin{array}{c}181.90 \pm \\
111.97^{\mathrm{a}}\end{array}$ & $\begin{array}{c}2.94 \pm \\
0.50^{\mathrm{b}}\end{array}$ & $\begin{array}{c}2.15 \pm \\
0.27^{\mathrm{a}}\end{array}$ & $\begin{array}{l}11.62 \pm \\
11.45^{\mathrm{a}}\end{array}$ & $\begin{array}{l}0.735^{ \pm} \\
0.155^{\mathrm{a}}\end{array}$ & $\begin{array}{l}3.46 \pm \\
0.32^{\mathrm{a}}\end{array}$ \\
\hline \multirow{2}{*}{ Day 2} & Post-C & $\begin{array}{c}4.20 \pm \\
1.45^{\mathrm{a}}\end{array}$ & $\begin{array}{c}27.66 \pm \\
7.42^{\mathrm{a}}\end{array}$ & $\begin{array}{c}10.01 \pm \\
3.55^{\mathrm{a}}\end{array}$ & $\begin{array}{c}255167 \pm \\
92500^{\mathrm{a}^{*}}\end{array}$ & $\begin{array}{c}167.25^{ \pm} \\
54.33^{\mathrm{a}}\end{array}$ & $\begin{array}{l}4.17 \pm \\
1.06^{\mathrm{c}^{* *}}\end{array}$ & $\begin{array}{r}2.41 \pm \\
0.25^{\mathrm{a}}\end{array}$ & $\begin{array}{c}7.06 \pm \\
1.38^{\mathrm{a}}\end{array}$ & $\begin{array}{c}0.716 \pm \\
0.104^{\mathrm{a}}\end{array}$ & $\begin{array}{l}4.10 \pm \\
0.67^{\mathrm{a} *}\end{array}$ \\
\hline & Post-HD & $\begin{array}{c}3.44 \pm \\
1.10^{\mathrm{a}}\end{array}$ & $\begin{array}{c}23.83 \pm \\
8.99^{\mathrm{a}}\end{array}$ & $\begin{array}{l}8.15 \pm \\
2.79^{\mathrm{a}}\end{array}$ & $\begin{array}{c}140150 \pm \\
59512^{b}\end{array}$ & $\begin{array}{c}111.17 \pm \\
70.40^{\mathrm{a}}\end{array}$ & $\begin{array}{l}2.39 \pm \\
0.83^{d}\end{array}$ & $\begin{array}{c}2.13 \pm \\
0.28^{\mathrm{a}}\end{array}$ & $\begin{array}{l}5.57 \pm \\
2.56^{\mathrm{a}}\end{array}$ & $\begin{array}{c}0.843 \pm \\
0.170^{\mathrm{a}}\end{array}$ & $\begin{array}{l}3.21 \pm \\
0.49^{\mathrm{b}}\end{array}$ \\
\hline \multirow{2}{*}{ Day 3} & Post-C & $\begin{array}{c}3.95 \pm \\
1.78^{\mathrm{a}}\end{array}$ & $\begin{array}{c}27.20 \pm \\
12.03^{\mathrm{a}}\end{array}$ & $\begin{array}{r}9.64 \pm \\
4.27^{\mathrm{a}}\end{array}$ & $\begin{array}{c}270250 \pm \\
117236^{\mathrm{a}}\end{array}$ & $\begin{array}{c}160.23 \pm \\
32.96^{\mathrm{a}^{*}}\end{array}$ & $\begin{array}{l}3.94 \pm \\
1.07^{\mathrm{a}^{*}}\end{array}$ & $\begin{array}{l}2.50 \pm \\
0.31^{\mathrm{a}^{*}}\end{array}$ & $\begin{array}{l}7.26 \pm \\
2.03^{\mathrm{a}}\end{array}$ & $\begin{array}{l}0.641 \pm \\
0.129^{\mathrm{a}^{*}}\end{array}$ & $\begin{array}{l}3.83 \pm \\
0.38^{\mathrm{a}}\end{array}$ \\
\hline & Post-HD & $\begin{array}{c}3.45 \pm \\
1.01^{\mathrm{a}}\end{array}$ & $\begin{array}{c}23.20 \pm \\
6.94^{\mathrm{a}}\end{array}$ & $\begin{array}{l}7.90 \pm \\
2.64^{\mathrm{a}}\end{array}$ & $\begin{array}{c}131120 \pm \\
71682^{\mathrm{a}}\end{array}$ & $\begin{array}{c}100.02 \pm \\
31.21^{\mathrm{b}}\end{array}$ & $\begin{array}{l}2.46 \pm \\
0.87^{\mathrm{b}}\end{array}$ & $\begin{array}{l}2.02 \pm \\
0.32^{b}\end{array}$ & $\begin{array}{c}5.98 \pm \\
1.36^{\mathrm{a}}\end{array}$ & $\begin{array}{c}0.844 \pm \\
0.123^{\mathrm{b}}\end{array}$ & $\begin{array}{l}3.60 \pm \\
0.58^{\mathrm{a}}\end{array}$ \\
\hline \multirow{2}{*}{ Day 4} & Post-C & $\begin{array}{r}4.29 \pm \\
1.35^{\mathrm{a}}\end{array}$ & $\begin{array}{c}30.40 \pm \\
8.62^{\mathrm{a}}\end{array}$ & $\begin{array}{c}10.66 \pm \\
3.40^{\mathrm{a}}\end{array}$ & $\begin{array}{c}299500 \pm \\
161544^{\mathrm{a}}\end{array}$ & $\begin{array}{c}157.93 \pm \\
33.85^{\mathrm{a}^{*}}\end{array}$ & $\begin{array}{l}4.39 \pm \\
0.94^{\mathrm{a}^{*}}\end{array}$ & $\begin{array}{c}2.46 \pm \\
0.34^{\mathrm{a}}\end{array}$ & $\begin{array}{l}7.26 \pm \\
2.32^{\mathrm{a}}\end{array}$ & $\begin{array}{c}0.830 \pm \\
0.121^{\mathrm{a}}\end{array}$ & $\begin{array}{r}4.03 \pm \\
0.28^{\mathrm{a}}\end{array}$ \\
\hline & Post-HD & $\begin{array}{c}3.21 \pm \\
0.93^{\mathrm{a}}\end{array}$ & $\begin{array}{c}22.20 \pm \\
6.14^{\mathrm{a}}\end{array}$ & $\begin{array}{l}7.54 \pm \\
2.12^{\mathrm{a}}\end{array}$ & $\begin{array}{c}227795 \pm \\
157929^{a}\end{array}$ & $\begin{array}{c}100.20 \pm \\
26.25^{b}\end{array}$ & $\begin{array}{c}2.84 \pm \\
0.89^{b}\end{array}$ & $\begin{array}{c}2.12 \pm \\
0.21^{\mathrm{a}}\end{array}$ & $\begin{array}{l}7.20 \pm \\
2.45^{\mathrm{a}}\end{array}$ & $\begin{array}{c}0.820 \pm \\
0.193^{\mathrm{a}}\end{array}$ & $\begin{array}{l}3.82 \pm \\
0.84^{\mathrm{a}}\end{array}$ \\
\hline \multirow{2}{*}{ Day 5} & Post-C & $\begin{array}{l}4.30 \pm \\
2.04^{\mathrm{a}}\end{array}$ & $\begin{array}{c}30.00 \pm \\
12.98^{\mathrm{a}}\end{array}$ & $\begin{array}{c}10.38 \pm \\
4.89^{\mathrm{a}}\end{array}$ & $\begin{array}{c}269000 \pm \\
195095^{\mathrm{a}}\end{array}$ & $\begin{array}{c}168.90 \pm \\
27.96^{\mathrm{a}}\end{array}$ & $\begin{array}{c}4.14 \pm \\
1.68^{\mathrm{a}}\end{array}$ & $\begin{array}{l}2.62 \pm \\
0.64^{\mathrm{a}}\end{array}$ & $\begin{array}{l}7.44 \pm \\
2.01^{\mathrm{a}}\end{array}$ & $\begin{array}{c}0.752 \pm \\
0.157^{\mathrm{a}}\end{array}$ & $\begin{array}{l}3.88 \pm \\
0.62^{\mathrm{a}}\end{array}$ \\
\hline & Post-HD & $\begin{array}{r}2.47 \pm \\
0.61^{\mathrm{a}}\end{array}$ & $\begin{array}{c}16.66 \pm \\
3.78^{\mathrm{a}}\end{array}$ & $\begin{array}{c}5.66 \pm \\
1.50^{\mathrm{a}}\end{array}$ & $\begin{array}{c}185808 \pm \\
119675^{\mathrm{a}}\end{array}$ & $\begin{array}{c}110.67 \pm \\
75.95^{\mathrm{a}}\end{array}$ & $\begin{array}{c}2.56 \pm \\
0.98^{\mathrm{a}}\end{array}$ & $\begin{array}{r}2.03 \pm \\
0.25^{\mathrm{a}}\end{array}$ & $\begin{array}{l}7.23 \pm \\
1.29^{\mathrm{a}}\end{array}$ & $\begin{array}{c}0.843 \pm \\
0.085^{\mathrm{a}}\end{array}$ & $\begin{array}{l}3.63 \pm \\
0.97^{\mathrm{a}}\end{array}$ \\
\hline
\end{tabular}

RBC's - Red blood cels; Ht - Hematocrit; Hb - Hemoglobin; sCr - Serum creatinine; sAlb - Serum albumin; P - Phosphorus; iCa ${ }^{+2}-$ Ionized calcium; $\mathrm{K}^{+}$- Potassium. Different letters represent statistically significant differences over time in the same treatment; $* P<0.05 \mathrm{x}$ Post; $* * P<0.01 \times$ Post.

the number of red blood cells and thus, hematocrit. Dogs submitted to IHD may develop anemia due to the dialyzer type, excessive negative access pressure, repeated blood sampling, and circuit clotting. Furthermore, prolonged anticoagulation can induce or exacerbate bleeding; hemolysis can also occur due to contaminants in the water supply, hypotonic or overheated dialysate, and extracorporeal circuit tubing problems (LANGSTON et al., 2017). In contrast, although there was a small reduction of RBC in CG dogs at the end of fluid therapy treatment, it was not statistically significant, demonstrating that fluid therapy does not increase anemia in patients with CKD.

The incidence of thrombocytopenia is commonly reported at the end of the hemodialysis sessions. Platelet count in HDG was significantly lower in POST samples compared with the PRE values at Day
5 (Table 1). Thrombocytopenia is a common adverse reaction observed in patients receiving heparin therapy, which results from blood contact with the dialysis membrane (ROSS, 2011). Comparisons between groups revealed slightly lower platelet count values in HDG than in $\mathrm{CG}$, with a statistically significant reduction observed in HDG on Day 2 (Table 2). As cited above, clotting in the extracorporeal circuit and dialyzer can reduce the number of platelets; likewise, uremic thrombocytopathy may also contribute to platelet loss (LANGSTON et al., 2017).

In CG, serum urea was significantly reduced in the POST sample compared with that in the PRE sample on Days 12 and 15. Urea and serum creatinine values were reduced in POST samples at other times, but the differences in serum creatinine were not statistically significant (Table 3 ). This is a 
Table 3 - Clinical values (average \pm standard deviation) of serum urea $\left(\mathrm{mg} \mathrm{dL}^{-1}\right)$, creatinine $\left(\mathrm{mg} \mathrm{dL}^{-1}\right)$, albumin $\left(\mathrm{g} \mathrm{dL}^{-1}\right)$, phosphorus $\left(\mathrm{mg}^{-1}\right.$ $\left.\mathrm{dL}^{-1}\right)$, ionic calcium $\left(\mathrm{mmol} \mathrm{L} \mathrm{L}^{-1}\right)$, and potassium $\left(\mathrm{mEq} \mathrm{L}^{-1}\right)$ of animals in the control group (CG) and the hemodialysis group (HDG) before (Pre) and after (Post) treatment (Day 1 to Day 15).

\begin{tabular}{|c|c|c|c|c|c|c|c|c|c|c|c|}
\hline \multicolumn{2}{|c|}{ Parameters } & \multicolumn{2}{|c|}{--------Day 1-------- } & \multicolumn{2}{|c|}{--------Day 5-------- } & \multicolumn{2}{|c|}{--------Day 8-------- } & \multicolumn{2}{|c|}{---------Day 12-------- } & \multicolumn{2}{|c|}{--------Day 15-------- } \\
\hline & & $\begin{array}{c}C G \\
(n=6)\end{array}$ & $\begin{array}{l}\text { HDG } \\
(\mathrm{n}=6)\end{array}$ & $\begin{array}{c}C G \\
(n=6)\end{array}$ & $\begin{array}{l}\text { HDG } \\
(n=6)\end{array}$ & $\begin{array}{c}C G \\
(n=6)\end{array}$ & $\begin{array}{l}\text { HDG } \\
(n=5)\end{array}$ & $\begin{array}{c}C G \\
(n=6)\end{array}$ & $\begin{array}{l}\text { HDG } \\
(n=5)\end{array}$ & $\begin{array}{c}C G \\
(n=5)\end{array}$ & $\begin{array}{l}\text { HDG } \\
(n=3)\end{array}$ \\
\hline \multirow{2}{*}{ Urea } & Pre & $\begin{array}{c}188.03 \pm \\
76.74^{\mathrm{a}}\end{array}$ & $\begin{array}{l}276.30 \pm \\
140.98^{\mathrm{c}^{* * *}}\end{array}$ & $\begin{array}{c}179.10 \pm \\
65.22^{\mathrm{a}}\end{array}$ & $\begin{array}{l}231.25 \pm \\
140.50^{\mathrm{a}^{*}}\end{array}$ & $\begin{array}{c}163.58 \pm \\
29.58^{\mathrm{a}}\end{array}$ & $\begin{array}{c}255.38 \pm \\
71.30^{\mathrm{a}^{*}}\end{array}$ & $\begin{array}{l}168.65 \pm \\
34.79^{c^{* *}}\end{array}$ & $\begin{array}{c}187.50 \pm \\
34.63^{\mathrm{a}^{*}}\end{array}$ & $\begin{array}{l}182.72 \pm \\
28.11^{\mathrm{a}^{*}}\end{array}$ & $\begin{array}{c}210.67 \pm \\
35.0^{\mathrm{a}}\end{array}$ \\
\hline & Post & $\begin{array}{c}174.43 \pm \\
56.96^{\mathrm{a}}\end{array}$ & $\begin{array}{l}181.90 \pm \\
111.97^{\mathrm{d}}\end{array}$ & $\begin{array}{c}167.25 \pm \\
54.33^{\mathrm{a}}\end{array}$ & $\begin{array}{c}111.17 \pm \\
70.40^{\mathrm{b}}\end{array}$ & $\begin{array}{c}160.23 \pm \\
32.96^{\mathrm{a}}\end{array}$ & $\begin{array}{c}100.02 \pm \\
31.21^{\mathrm{b}}\end{array}$ & $\begin{array}{c}157.93 \pm \\
33.85^{\mathrm{d}}\end{array}$ & $\begin{array}{c}100.20 \pm \\
26.25^{\mathrm{b}}\end{array}$ & $\begin{array}{c}168.90 \pm \\
27.96^{\mathrm{b}}\end{array}$ & $\begin{array}{c}110.67 \pm \\
75.9^{\mathrm{a}}\end{array}$ \\
\hline \multirow{2}{*}{$\mathrm{sCr}$} & Pre & $\begin{array}{l}4.35 \pm \\
0.55^{\mathrm{a}}\end{array}$ & $\begin{array}{l}4.38 \pm \\
0.42^{\mathrm{c}^{* *}}\end{array}$ & $\begin{array}{l}4.14 \pm \\
0.59^{\mathrm{a}}\end{array}$ & $\begin{array}{c}5.02 \pm \\
0.72^{\mathrm{c}^{* *}}\end{array}$ & $\begin{array}{l}4.14 \pm \\
1.00^{\mathrm{a}}\end{array}$ & $\begin{array}{l}5.36 \pm \\
1.63^{\mathrm{a}^{*}}\end{array}$ & $\begin{array}{l}4.44 \pm \\
0.67^{\mathrm{a}}\end{array}$ & $\begin{array}{l}4.89 \pm \\
0.89^{\mathrm{c}^{* *}}\end{array}$ & $\begin{array}{l}4.18 \pm \\
1.28^{\mathrm{a}}\end{array}$ & $\begin{array}{l}5.44 \pm \\
0.90^{\mathrm{a}}\end{array}$ \\
\hline & Post & $\begin{array}{l}4.04 \pm \\
0.78^{\mathrm{a}}\end{array}$ & $\begin{array}{c}2.94 \pm \\
0.50^{\mathrm{d}}\end{array}$ & $\begin{array}{l}4.17 \pm \\
1.06^{\mathrm{a}}\end{array}$ & $\begin{array}{l}2.39 \pm \\
0.83^{\mathrm{d}}\end{array}$ & $\begin{array}{l}3.94 \pm \\
1.07^{\mathrm{a}}\end{array}$ & $\begin{array}{l}2.46 \pm \\
0.87^{\mathrm{b}}\end{array}$ & $\begin{array}{c}4.39 \pm \\
0.95^{\mathrm{a}}\end{array}$ & $\begin{array}{l}2.84 \pm \\
0.89^{d}\end{array}$ & $\begin{array}{l}4.14 \pm \\
1.68^{\mathrm{a}}\end{array}$ & $\begin{array}{c}2.56 \pm \\
0.98^{\mathrm{a}}\end{array}$ \\
\hline \multirow{2}{*}{ sAlb. } & Pre & $\begin{array}{l}2.53 \pm \\
0.25^{\mathrm{a}^{* * *}}\end{array}$ & $\begin{array}{c}2.35 \pm \\
0.40^{\mathrm{a}}\end{array}$ & $\begin{array}{c}2.48 \pm \\
0.26^{\mathrm{a}}\end{array}$ & $\begin{array}{l}2.38 \pm \\
0.32^{\mathrm{a}^{*}}\end{array}$ & $\begin{array}{c}2.56 \pm \\
0.38^{\mathrm{a}}\end{array}$ & $\begin{array}{l}2.38 \pm \\
0.41^{\mathrm{c} * *}\end{array}$ & $\begin{array}{l}2.60 \pm \\
0.34^{\mathrm{a}^{*}}\end{array}$ & $\begin{array}{l}2.32 \pm \\
0.43^{\mathrm{a}}\end{array}$ & $\begin{array}{c}2.82 \pm \\
0.59^{\mathrm{a}}\end{array}$ & $\begin{array}{c}2.26 \pm \\
0.30^{\mathrm{a}}\end{array}$ \\
\hline & Post & $\begin{array}{l}2.31 \pm \\
0.24^{\mathrm{b}}\end{array}$ & $\begin{array}{l}2.15 \pm \\
0.27^{\mathrm{a}}\end{array}$ & $\begin{array}{c}2.41 \pm \\
0.25^{\mathrm{a}}\end{array}$ & $\begin{array}{l}2.13 \pm \\
0.28^{\mathrm{b}}\end{array}$ & $\begin{array}{c}2.50 \pm \\
0.31^{\mathrm{a}}\end{array}$ & $\begin{array}{l}2.02 \pm \\
0.32^{\mathrm{d}}\end{array}$ & $\begin{array}{c}2.46^{ \pm} \\
0.34^{\mathrm{b}}\end{array}$ & $\begin{array}{l}2.12 \pm \\
0.21^{\mathrm{a}}\end{array}$ & $\begin{array}{l}2.62 \pm \\
0.64^{\mathrm{a}}\end{array}$ & $\begin{array}{c}2.03 \pm \\
0.25^{\mathrm{a}}\end{array}$ \\
\hline \multirow[b]{2}{*}{$\mathrm{P}$} & Pre & $\begin{array}{l}8.16 \pm \\
2.71^{\mathrm{a}}\end{array}$ & $\begin{array}{l}20.48 \pm \\
16.27^{\mathrm{a}^{*}}\end{array}$ & $\begin{array}{l}7.19 \pm \\
1.33^{\mathrm{a}}\end{array}$ & $\begin{array}{l}9.56 \pm \\
4.04^{\mathrm{a}^{*}}\end{array}$ & $\begin{array}{c}7.34 \pm \\
1.81^{\mathrm{a}}\end{array}$ & $\begin{array}{l}13.68 \pm \\
2.51^{\mathrm{c}^{* *}}\end{array}$ & $\begin{array}{l}7.82 \pm \\
2.77^{\mathrm{a}}\end{array}$ & $\begin{array}{c}11.26 \pm \\
5.70^{\mathrm{a}}\end{array}$ & $\begin{array}{l}7.70 \pm \\
2.77^{\mathrm{a}}\end{array}$ & $\begin{array}{c}16.10^{ \pm} \\
2.31^{\mathrm{a}}\end{array}$ \\
\hline & Post & $\begin{array}{l}6.86 \pm \\
2.38^{\mathrm{a}}\end{array}$ & $\begin{array}{l}11.61 \pm \\
11.45^{b}\end{array}$ & $\begin{array}{l}7.06 \pm \\
1.38^{\mathrm{a}}\end{array}$ & $\begin{array}{l}5.56 \pm \\
2.56^{\mathrm{b}}\end{array}$ & $\begin{array}{l}7.26 \pm \\
2.03^{\mathrm{a}}\end{array}$ & $\begin{array}{l}5.98 \pm \\
1.36^{\mathrm{d}}\end{array}$ & $\begin{array}{l}7.26 \pm \\
2.32^{\mathrm{a}}\end{array}$ & $\begin{array}{l}7.20 \pm \\
2.45^{\mathrm{a}}\end{array}$ & $\begin{array}{l}7.44 \pm \\
2.01^{\mathrm{a}}\end{array}$ & $\begin{array}{c}7.23 \pm \\
1.29^{\mathrm{a}}\end{array}$ \\
\hline \multirow{2}{*}{$\mathrm{iCa}^{+2}$} & Pre & $\begin{array}{c}0.818 \pm \\
0.125^{\mathrm{a}}\end{array}$ & $\begin{array}{c}0.750 \pm \\
0.187^{\mathrm{a}}\end{array}$ & $\begin{array}{c}0.861 \pm \\
0.145^{\mathrm{a}}\end{array}$ & $\begin{array}{c}0.860 \pm \\
0.376^{\mathrm{a}}\end{array}$ & $\begin{array}{c}0.715 \pm \\
0.198^{\mathrm{a}}\end{array}$ & $\begin{array}{c}0.786 \pm \\
0.175^{\mathrm{a}}\end{array}$ & $\begin{array}{c}0.713 \pm \\
0.167^{\mathrm{a}}\end{array}$ & $\begin{array}{c}0.890 \pm \\
0.272^{\mathrm{a}}\end{array}$ & $\begin{array}{c}0.748 \pm \\
0.152^{\mathrm{a}}\end{array}$ & $\begin{array}{c}0.833 \pm \\
0.275^{\mathrm{a}}\end{array}$ \\
\hline & Post & $\begin{array}{c}0.764 \pm \\
0.092^{\mathrm{a}}\end{array}$ & $\begin{array}{c}0.735 \pm \\
0.155^{\mathrm{a}}\end{array}$ & $\begin{array}{c}0.716 \pm \\
0.104^{\mathrm{a}}\end{array}$ & $\begin{array}{c}0.843 \pm \\
0.170^{\mathrm{a}}\end{array}$ & $\begin{array}{l}0.641 \pm \\
0.129^{\mathrm{a}}\end{array}$ & $\begin{array}{l}0.844 \pm \\
0.123^{\mathrm{a}}\end{array}$ & $\begin{array}{c}0.830 \pm \\
0.121^{\mathrm{a}}\end{array}$ & $\begin{array}{c}0.820 \pm \\
0.193^{\mathrm{a}}\end{array}$ & $\begin{array}{c}0.752 \pm \\
0.157^{\mathrm{a}}\end{array}$ & $\begin{array}{c}0.843 \pm \\
0.085^{\mathrm{a}}\end{array}$ \\
\hline \multirow{2}{*}{$\mathrm{K}^{+}$} & Pre & $\begin{array}{c}4.07 \pm \\
0.70^{\mathrm{a}}\end{array}$ & $\begin{array}{c}3.71 \pm \\
0.65^{\mathrm{a}}\end{array}$ & $\begin{array}{l}4.33 \pm \\
0.56^{\mathrm{a}}\end{array}$ & $\begin{array}{l}3.73 \pm \\
0.87^{\mathrm{a}}\end{array}$ & $\begin{array}{l}4.23 \pm \\
0.59^{\mathrm{a}^{*}}\end{array}$ & $\begin{array}{l}4.26 \pm \\
0.98^{\mathrm{a}}\end{array}$ & $\begin{array}{l}4.15 \pm \\
0.62^{\mathrm{a}}\end{array}$ & $\begin{array}{l}4.68 \pm \\
1.00^{\mathrm{a}}\end{array}$ & $\begin{array}{c}3.96 \pm \\
0.51^{\mathrm{a}}\end{array}$ & $\begin{array}{l}4.80 \pm \\
1.51^{\mathrm{a}}\end{array}$ \\
\hline & Post & $\begin{array}{c}4.06 \pm \\
0.59^{\mathrm{a}}\end{array}$ & $\begin{array}{l}3.47 \pm \\
0.32^{\mathrm{a}}\end{array}$ & $\begin{array}{c}4.10 \pm \\
0.6^{\mathrm{a}}\end{array}$ & $\begin{array}{l}3.21 \pm \\
0.49^{\mathrm{a}}\end{array}$ & $\begin{array}{l}3.83 \pm \\
0.39^{\mathrm{b}}\end{array}$ & $\begin{array}{l}3.60 \pm \\
0.58^{\mathrm{a}}\end{array}$ & $\begin{array}{l}4.03 \pm \\
0.28^{\mathrm{a}}\end{array}$ & $\begin{array}{l}3.82 \pm \\
0.84^{\mathrm{a}}\end{array}$ & $\begin{array}{l}3.88 \pm \\
0.62^{\mathrm{a}}\end{array}$ & $\begin{array}{l}3.63 \pm \\
0.97^{\mathrm{a}}\end{array}$ \\
\hline
\end{tabular}

$\mathrm{sCr}$ - Serum creatinine; sAlb - Serum albumin; P - Phosphorus; iCa ${ }^{+2}$ - Ionized calcium; $\mathrm{K}^{+}-$Potassium. Different letters represent statistical difference over time in the same treatment; ${ }^{*} P<0.05 \mathrm{x}$ Post; ${ }^{* *} P<0.01 \mathrm{x}$ Post.

common finding in IHD treatment, which uses this parameter to monitor the efficiency and efficacy of hemodialysis treatment (COWGILL \& FRANCEY, 2012). Although, urea and serum creatinine levels were clearly lower in HDG compared with the levels in CG POST treatment, it is important to notice that PRE treatment, both groups had similar values showing that significant reductions in serum urea and creatinine are common in the IHD for dogs with CKD stage III, but this reduction only occurs for a short period of time (Table 2).

In this study, most of the dogs had higher serum phosphorus levels than the recommended target level in CKD stage 3 (FOSTER, 2016). As reported for urea and creatinine concentrations, phosphorus also decreases with the prescription of hemodialysis (COWGILL \& FRANCEY, 2012). Phosphorus reduction in both groups can be explained by the prescription of oral phosphate binders; however, this reduction was not statistically significant in CG due to the short duration of the treatment, requiring a longer period of continuous use. In contrast, HDG had a marked reduction in all POST samples with statistical significance on Day 1, Day 5, and Day 8 (Table 3). In contrast, ionized calcium levels in dogs from both groups were below the references values of $1.25-1.45 \mathrm{mmol} \mathrm{L}^{-1}$ (STOCKHAM \& SCOTT, 2002). The use of heparin is well known to cause false decreases in calcium, possibly due to the technique (plasma analytes), 
dilution, and binding effects of excess heparin; premeasured lyophilized heparin is recommended for blood gas and ionized calcium measurement (GORMAN, 2017).

There were no significant changes in albumin levels after hemodialysis or fluid therapy except on PRE and POST samples on Day 1 and Day 12 of CG and on Day 5 and Day 8 of HDG (Table 3). When the two groups were compared, serum albumin levels were significantly reduced after hemodialysis treatment on Day 8 (Table 3 ). However, neither therapy demonstrated a significant decrease in the serum concentration of albumin during treatment.

All dogs from both groups $(n=12)$ were sub staged as proteinuric; consequently, they were treated with an ACEI as part of the clinical treatment (BARTGES, 2012; IRIS, 2015). Further studies are needed to correlate serum albumin levels and dialysis time; yet, we suspect that the shortprescribed time of the IHD session (120 minutes) may have contributed to a better control of serum albumin levels in HDG.

Twenty-five sessions of hemodialysis in six dogs were performed in this study, and few complications were observed. Discomfort and vocalization were observed in five sessions (20\%); this was the most frequent complication observed. Emesis and diarrhea occurred in one session (4\%). Loss of vascular access and a subsequent requirement for double lumen catheter change occurred in one session (4\%). Although, IHD is a more efficient technique for the removal of nitrogenous end products than fluid therapy, this extracorporeal technique demonstrates a degree of complexity that can lead to several complications when compared with fluid therapy. The success of this treatment modality will rely on the active cooperation of a well-trained veterinarian and technical staff.

Dogs from both groups had a similar survival period, and no significant difference in survival was observed $(P=0.2980)$. When the survival rates in both groups were evaluated, GHD and $C G$ revealed the same death incidence during treatment prescription (33\% from each group). Yet, when survival was evaluated in both groups over 60 days, the $\mathrm{CG}$ had a death incidence of $83 \%$, with an average survival of $48.5 \pm 18.6$ days, while the HDG had a death incidence of $100 \%$, with an average survival of $28 \pm 13.8$ days (Figure 1). Therefore, we do not encourage early prescription of intermittent hemodialysis as a therapy modality for dogs with CKD in stage III.

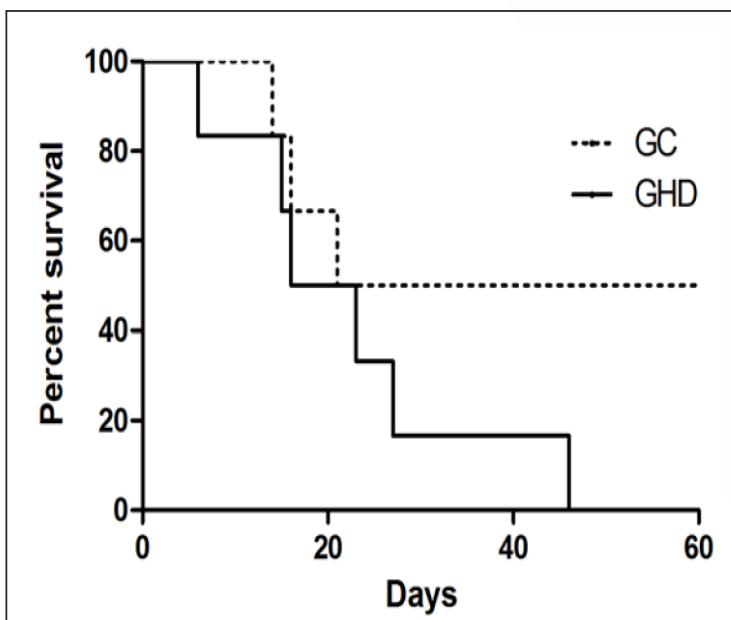

Figure 1 - Survival (days) of dogs in the control group (CG) and the hemodialysis group (HDG) during two months (60 days) after the treatment started.

\section{CONCLUSION}

The prescription of intermittent hemodialysis in this study did not change the survival rate of dogs with chronic kidney disease in stage III. However, IHD did improve overall blood chemistry by reducing the levels of serum urea, creatinine, and phosphorus more effectively than intravenous fluid therapy alone. Although, dialysis treatment was more invasive and contained greater risks than treatment with intravenous fluid therapy, the incidence of complications from IHD was low in this study, indicating that it is a safe technique to prescribe for dogs with CKD in stage III. However, it did not increase patient survival. Therefore, this study does not support the early prescription of IHD for dogs with chronic kidney disease stage III.

\section{ACKNOWLEDGMENT}

The project was approved by the Comissão de Ética no Uso de Animais (CEUA) on February 8, 2013 (file n $^{\circ}$ 18/2013 of CEUA). The authors are grateful for the financial support of the Fundação de Amparo a Pesquisa do Estado de São Paulo (FAPESP) (Process: 2013-02932-8).

\section{REFERENCES}

BARTGES, J.W. Chronic kidney disease in dogs and cats. Veterinary Clinics Small Animal Practice, v.42, p.669-692, 2012. Available from: $<$ http://www.vetsmall.theclinics.com/article/S01955616(12)00072-1/abstract>. Accessed: Mar 22, 2015. doi: 10.1016/j. cvdm.2012.04.008.

BLOOM, C.A.; LABATO, M.A. Intermittent hemodialysis for small animals. Veterinary Clinics Small Animal Practice, v.41, 
n.1, p.115-133, 2011. Available from: <http://www.ncbi.nlm.nih. gov/pubmed/21251513>. Accessed: Jan. 25, 2014. doi: 10.1016/j. cvsm.2010.11.001.

COWGILL, L.D.; FRANCEY, T. Hemodialysis and extracorporeal blood purification. In: DIBARTOLA, S.P. Fluid, eletrolyte and acid-base disorders in small animal practice. 4.ed. Missouri: Saunders Elsevier, 2012. Chap.29, p.680-713.

COWGILL, L.D.; GUILLAUMIN, J. Extracorporeal renal replacement therapy and blood purification in critical care. Journal of Veterinary Emergency and Critical Care, v.23, n.2, p.1-11, 2013. Available from: <http://www.ncbi.nlm.nih.gov/pubmed/23517350>. Accessed: Mar. 22, 2015. doi: 10.1111/vec.12028.

COWGILL, L.D. Urea kinetics and intermittent dialysis prescription in small animals. Veterinary Clinics Small Animal Practice, v.41, p.193-225, 2011. Available from: <http://www. ncbi.nlm.nih.gov/pubmed/21251518>. Accessed: Jan. 25, 2014. doi: $10.1016 /$ j.cvsm.2010.12.002.

DIBARTOLA, S.P.; BATEMAN, S. Introduction to fluid therapy. In: DIBARTOLA, S.P. Fluid, eletrolyte and acid-base disorders in small animal practice. 4.ed. Missouri: Saunders Elsevier, 2012. Chap.14, p.331-350.

FALCO, S. Thromboelastometry used for evaluation of blood coagulability in dogs with kidney diseases. Acta Veterinaria Brno, v.82, p.209-214, 2013. Available from: <https://actavet.vfu. cz/media/pdf/avb_2013082020209.pdf >. Accessed: Mar. 16, 2016. doi: $10.2754 /$ avb201382020209.

FISCHER, J.R. et al. Veterinary hemodialysis: advances in management and technology. Veterinary Clinics of North America: Small Animal Practice, v.34, p.935-967, 2004. Available from: $<$ http://www.ncbi.nlm.nih.gov/pubmed/15223210>. Accessed: Feb. 12, 2014. doi: 10.1016/j.cvsm.2004.03.007.

FOSTER, J.D. Update on mineral and bone disorder in chronic kidney disease. Veterinary Clinics of North America: Small Animal Practice, v.46, p.1131-1149, 2016. Available from: <http:// www.vetsmall.theclinics.com/article/S0195-5616(16)30039-0/ pdf $>$. Accessed: Oct. 10, 2016. doi: 10.1016/j.cvsm.2016.06.003.

GORMAN, E. Spurious results in mineral and electrolyte analysis. Veterinary Clinics of North America: Small Animal Practice, v.47, p.263-272, 2017. Available from: <http://www.sciencedirect.
com/science/article/pii/S0195561616301371>. Accessed: May 10, 2017. doi: $10.1016 /$ j.cvsm.2016.10.019.

IRIS Staging of CKD. 2015. Available from: < http://www.iriskidney.com/ guidelines/en/StaginCGkd.shtml>. Online. Accessed: Feb. 05, 2016.

JOHNSON, R.A.; WICHERN, D.N. Applied multivariate statistical analysis. 5.ed. New Jersey: Practice-Hall, 2001. 767 p.

KOMENO, M. et al. Role of nitric oxide in hemodialysis-related hypotension in an experimental renal dysfunction dog model. Journal of Veterinary Medicine Science, v.66, p.53-57, 2004. Available from: $<$ http://www.ncbi.nlm.nih.gov/pubmed/14960811>. Accessed: Nov. 17, 2014.

LANGSTON, C. et al. Blood transfusions in dogs and cats receiving hemodialysis: 230 cases (June 1997-September 2012). Journal of Veterinary Internal Medicine, v.31, n.2, p.402409, 2017. Available from: <https://www.ncbi.nlm.nih.gov/pmc/ articles/PMC5354009/>. Accessed: May 09, 2017. doi: 10.1111/ jvim. 14658 .

POLZIN, D.J. Chronic kidney disease in small animals. Veterinary Clinics Small Animal Practice, v.41, n.1, p.15-30, 2011. Available from: <http://www.sciencedirect.com/science/journal/01955616/41/1>. Accessed: Mar. 15, 2016. doi: 10.1016/j.cvsm.2010.09.004.

POLZIN, D.J. Evidence-based step-wise approach to managing chronic kidney disease in dogs and cats. Journal of Veterinary Emergency and Critical Care, v.23, n.2, p.205-215, 2013. Available from: $<$ http://www.ncbi.nlm.nih.gov/pubmed/23470210>. Accessed: Nov. 17, 2014. doi: 10.1111/vec.12034.

ROSS, S. Anticoagulation in intermittent hemodialysis: pathways, protocols, and pitfalls. Veterinary Clinics Small Animal Practice, v.41, n.1, p.163-175, 2011. Available from: <http://www.ncbi.nlm. nih.gov/pubmed/21251516>. Accessed: Jan. 25, 2014. doi: 10.1016/j. cvsm.2010.12.001.

STOCKHAM, S.L.; SCOTT, M.A. Fundamentals of veterinary clinical pathology. Iowa: Iowa State University, 2002. 920 p.

WAKI, M.F. et al. Classification into stages of chronic kidney disease in dogs and cats - clinical, laboratorial and therapeutic approach. Ciência Rural, v.40, n.10, p.2226-2234, 2010. Available from: $<$ http://www.scielo.br/pdf/cr/v40n10/a741cr3532.pdf>. Accessed: Dec. 21, 2014. doi: 10.1590/S0103-84782010005000168. 